\title{
Current Advancements on the Significance of Oncolytic Viruses in the Treatment of Tumor Cells
}

\section{Addisu Demeke*, Asmelash Tassew and Aklilu Feleke}

Department of Microbiology, Immunology and Public Health, College of Veterinary Medicine and Agriculture, Addis Ababa University, Debre Zeit, Ethiopia

*Corresponding author: Addisu Demeke, Department of Microbiology, Immunology and Public Health, College of Veterinary Medicine and Agriculture, Addis Ababa University, Debre Zeit, Ethiopia, Tel: +251-111-239746; E-mail: ademeke16@yahoo.com

Rec date: Dec 28, 2015; Acc date: Feb 04, 2016; Pub date: Feb 06, 2016

Copyright: $\odot 2016$ Demeke A, et al. This is an open-access article distributed under the terms of the Creative Commons Attribution License, which permits unrestricted use, distribution, and reproduction in any medium, provided the original author and source are credited.

\begin{abstract}
The occurrence and severity of tumor, which is caused by many factors and etiological agents, become rapid and its distribution is worldwide that lead to death of many individuals. In order to avoid associated complications scientists discovered a means for the removal of tumor using oncolytic virus called virotherapy. This review is aiming to give highlight about the current advancements on the significance of oncolytic viruses in the treatment of tumor cells. Oncolytic viruses carry the promise to efficiently target cancer cells for destruction and spread throughout tumor tissue to reach distant loci without causing collateral damage to healthy tissues. Current oncolytic virotherapy strategies are based on the common molecular mechanisms of viral infection and cell transformation, like apoptotic dysregulation and cell cycle disruption. In this regards this time scientists is being done active research to improve the accessibility, safety and efficacy of oncolytic virotherapy.
\end{abstract}

Keywords: Oncolytic virotherapy; Oncolytic viruses; Tumor cells

\section{Introduction}

Currently, the spread and severity of cancer increase very rapidly and the complex nature of this disease which often involves multiple mutations, overlapping signaling pathways, and the ability to adapt and develop resistance to various therapeutics becomes more evident. Such a complex disease necessitates equally complex therapies such as oncolytic viruses [1].

Over the past 40 years, anticancer agents have entered clinical practice and improved survival for many patients with cancer. Unfortunately, even for patients who achieve remission following treatment with surgery, chemotherapy, radiation therapy, and some of the newer targeted inhibitors and antibodies, relapse often occurs months or years later. Hypotheses to explain this phenomenon include ideas such as tumor cell acquisition of resistance, suboptimal surgical debulking and inability of chemotherapy and radiotherapy to target all cancer cells within a given patient. The cancer stem cell (CSC) theory postulates that there are subpopulations of cells, also known as cancerinitiating cells (CICs), responsible for relapse. "CSCs" was originally coined to describe features of these cells that are similar to bona fide normal stem cells [2].

The past decade has seen an explosion of research into the field of gene therapy and therapeutic or so called "oncolytic," viruses. Oncolytic virus therapy is the approach in which viruses replicate specifically in cancer cells and ultimately kill them. These viruses are either natural or genetically engineered from different human and animal viruses including HSV-1, Adenovirus, Reovirus, and New Castle Disease Virus [3]. These agents hold much promise as they have been shown to be efficacious against malignant tissues, yet minimally toxic to their normal cell and tissue counterparts. Oncolytic viruses are effective against a wide variety of human cancers in preclinical models and encouraging results from clinical trials are beginning to accumulate [4].
Oncolytic virotherapy is undergoing promising new research as a cancer treatment strategy, especially in conjunction with established therapies such as radiation. The ability of intravenously (IV) delivered oncolytic viruses to express antitumor genes, as well as to directly destroy cancer cells, makes them potentially effective against malignant cancers, although efficacy is currently still limited [5].

Therefore the objective of this seminar paper is to describe the current advancements on the significance of oncolytic viruses in the treatment of tumor cells.

\section{Virotherapeutics}

\section{Basis and advances of virotherapeutics}

Since the discovery of viruses in the nineteenth century, scientists have seen their potential in the treatment of cancer. The impetus for the development was the observation that cancer patients underwent regression after acquiring a viral infection. The first clinical trials involved transmitting bodily fluids containing viral particles to other cancer patients and observing the result. In these studies, clinicians used newly discovered viruses without knowing their biological behavior. Likely, the patients' immune systems destroyed the viruses, removing them from the body so that the virus failed to destroy the malignant cell growth. With the development of new models and methods for virus replication in the 1950's and 1960's, there were many attempts to develop viruses with greater tumor specificity, but many researchers stopped working on development due to limited success [6].

For several years, research in this field was inadequate due to technological limitations. After the 1960's, oncolytic virotherapy was almost entirely abandoned due to the lack of clear and promising results from clinical trials; at that time, molecular methods were not as advanced, so oncolytic virotherapy was in danger of being abandoned altogether. Now, as genetic engineering has advanced so enormously 
and rapidly, it has revived interest in oncolytic virotherapy. An increased understanding of virology, as well as experience using viruses in cancer gene therapy, has prompted a new wave of oncolytic virotherapy [7].

Many different viruses are used in oncolytic virotherapy. The use of genetically engineered, tumor-targeting viruses as oncolytic agents recently has emerged as a promising new area for novel cancer therapies. The first viruses to enter the clinic, such as ONYX-015 (an oncolytic adenovirus), demonstrated both the safety and anti-tumor potential of this approach. The results of these early trials also have allowed investigators to examine the restrictions and limitations of these viruses, and to develop potentially far more effective approaches [8].

Oncolytic virus (OV) therapy is based on the concept of using live viruses to selectively infect and replicate in cancer cells, with minimal destruction of non-neoplastic tissue. While the concept of using live viruses to infect and destroy tumors dates back to nearly over a century, advances in molecular biology and virology have accelerated the development of OVs in the last two decades [9].

Conditionally replication competent viruses are genetically engineered or selected to be avirulent in normal cells but can exploit the aberrant molecular / genetic pathways in tumors resulting in viral replication and cancer cell lytic destruction. This leads to their efficient replication within cancer cells and the lytic destruction of the infected malignant cell. Oncolytic viruses derived from Herpes Simplex Virus-1 (HSV-1), Adenovirus (Ad), New Castle Disease Virus (NDV), and Reovirus (RV) have been tested in several clinical trials for the treatment of malignant glioma. In all of these trials intratumoral administration of infectious oncolytic virus particles was found to be safe but significant evidence of efficacy remains to be established [10].

Several innovative strategies to enhance intratumoral viral spread and antitumor efficacy without compromising its safety are currently under investigation. These studies have resulted in the development of several patients which may contribute towards the advancement in future therapeutics [11].

\section{Characteristics of oncolytic viruses}

Oncolytic viruses are defined as those viruses that are capable of specifically targeting, and subsequently destroying tumor cells without causing excessive damage to surrounding normal tissues. These viruses are able to replicate in the target tumor cells, thereby producing high levels of infectious progeny virus and subsequently enabling the infection of additional malignant cells to be employed successfully and safely as an anti-cancer therapeutic. It is desired that an oncolytic virus displays a number of attributes: relatively low pathogenicity, able to replicate specifically in malignant cells, easily genetically manipulated, relatively well characterized in terms of viral genome and protein function, possess a rapid life cycle, well characterized in terms of mechanism of oncolytic action and tumor cell specificity, able to be delivered systemically, display susceptibility to an antiviral drug, and not cause serious side effects following administration. Some oncolytic viruses are naturally occurring, whilst others are genetically engineered to reduce pathogenicity, enhance tumour cell selectivity and encode therapeutic genes. Naturally occurring oncolytic viruses include Newcastle disease virus (NDV), Vesicular Stomatitis Virus (VSV), myxoma virus and reovirus. Engineered oncolytic viruses encompass backbones from adenoviruses, vaccina viruses and HSV [11].
Some viruses have been engineered with specific gene deletion in which these genes are crucial for the survival of viruses in normal cells but expendable in cancer cells. Deletion of the gene that encodes thymidine kinase, an enzyme needed for nucleic acid metabolism, results in dependence of viruses such as HSV and vaccinia virus on cellular thymidine kinase expression, which is high in proliferating cancer cells but not in normal cells [12].

\section{Cancer therapy using oncolytic viruses}

Viruses implemented in cancer gene therapy: Oncolytic viruses fall into broad categories: (i) wild-type animal viruses that do not typically infect human cells but are cytotoxic to human cancer cells, (ii) attenuated mutants of human viruses in which critical genes for virus replication that are dispensable in cancer cells have been deleted or mutated, and (iii) viruses that have been attenuated by serial passage in culture, such as most live virus vaccines (Table 1) [13].

Adenovirus: In order to replicate, adenovirus promotes entry of cells into the G1 phase of the cell cycle by binding $\mathrm{Rb}$ via the immediateearly protein E1A and releasing the transcriptional factor E2F. Thus, Ad is capable of infecting both dividing and non-dividing cells. Because many tumor cells harbor defects in the $\mathrm{Rb} / \mathrm{p} 16$ pathway, mutant Ads with a 24 base pair deletion of the E1A Rb binding site (24) showed tumor selectivity as viral replication was abrogated in normal cells with intact Rb/p16 [15].

Most Ad serotypes, including commonly used Ad5, enter into cells through their viral fiber knob binding to the host cell surface coxsackie-Ad receptor, which is highly expressed on normal epithelial cells but lacking in many tumor cells [16]. Modification of the viral capsid to change the Ad tropism has been a common strategy to overcome the lack of coxsackie-Ad receptor in tumor cells.

Eriksson et al. [17] showed capsid-modified E1A mutated Ads, Ad5/3-24, which uses the Ad serotype 3 receptor that is highly expressed in tumor cells, and Ad5. Pk7-24, which enters through heparan sulfate proteoglycans, was able to kill breast CICs. Breast CICs have been identified as a CD44+/ CD24-/low population in breast carcinoma [18].

Reovirus: Reovirus (respiratory enteric orphan virus) is a doublestranded RNA virus generally considered to be benign in humans, typically causing at most mild respiratory or gastrointestinal ailments. Interestingly, the virus is highly cytopathic for a variety of cultured cancer cell lines and induces rapid tumor regression in mouse xenograft models of a variety of human cancers including those from colon, ovarian, breast, lymphoma, brain and spinal cancer derived cells [19].

Reovirus is oncolytic in its naturally isolated state and over the past decade has been in development as a potential cancer therapeutic (Reolysin). After promising results from phase I/II clinical trials on various cancers, Reolysin is scheduled to begin phase III clinical trials for head and neck cancer in the 4th quarter of 2009 [20].

Recently they used biopsy cores taken from the primary tumor of a breast cancer patient to grow tumors in the mammary fat pad of immunocompromised mice. Subsequent reovirus injection induced regression of these patient-derived breast tumors [21].

$\mathrm{CIC}$ and non-CIC populations in the reovirus-treated tumors were found to be equally infected and eradicated. As predicted, it appears that characteristics of CICs that make them resistant to common 
Citation: Demeke A, Tassew A, Feleke A (2016) Current Advancements on the Significance of Oncolytic Viruses in the Treatment of Tumor

chemotherapeutics and radiation treatments are not hindering factors in reovirus cancer therapy [22].

\begin{tabular}{|c|c|c|c|}
\hline Genome & Family & Genus & Species / Strain / Vector \\
\hline \multicolumn{4}{|l|}{ DNA } \\
\hline ds & $\begin{array}{l}\text { Adenoviridae } \\
\text { Poxviridae }\end{array}$ & $\begin{array}{l}\text { Mastadenovirus } \\
\text { Aviadenovirus } \\
\text { Orthopoxvirus }\end{array}$ & $\begin{array}{l}\text { Ad serotype } 5 \text { and several derivatives in experimental settings and in clinical trials. } \\
\text { Conditionally replicating vectors based on canine adenovirus } \\
\text { Replication-deficient vector CELO } \\
\text { Replication-competent vectors based on Vaccinia Strains WR and Wyeth. }\end{array}$ \\
\hline ds & Parvoviridae & Parvovirus & $\begin{array}{l}\text { Live autonomous rodent parvovirus } \mathrm{H}-1 \text {. } \\
\text { Both live virus and replicons of minute virus mice. } \\
\text { Replicons of retargeted feline panleukopenia virus. }\end{array}$ \\
\hline \multicolumn{4}{|l|}{ RNA } \\
\hline ds & Reoviridae & Orhtoreovirus & $\begin{array}{l}\text { Live reovirus type } 3 \text { strain Dearing (T3D) in both pre-clinical } \\
\text { experiments and in clinical trials. }\end{array}$ \\
\hline ss & $\begin{array}{l}\text { Orthomyxoviridae } \\
\text { Paramyxomaviri } \\
\text { dae }\end{array}$ & $\begin{array}{l}\text { Influenza virus A } \\
\text { Avulavirus }\end{array}$ & $\begin{array}{l}\text { Replication-competent NS1 deleted influenza A. } \\
\text { Experimental therapy in several cancer models using live attenuated } \\
\text { Newcastle disease virus strains such as } 73-\mathrm{T} \text {, Ulster and Italian. } \\
\text { Clinical trials with the live attenuated PV701 and MTH-68/H strains of Newcastle } \\
\text { disease virus. }\end{array}$ \\
\hline \multicolumn{4}{|l|}{ DNA } \\
\hline ss & $\begin{array}{l}\text { Rhabdoviridae } \\
\text { Coronaviridae } \\
\text { Picarnaviridae } \\
\text { Togarviridae } \\
\text { Retroviridae }\end{array}$ & $\begin{array}{l}\text { Respirovirus } \\
\text { Rubulavirus } \\
\text { Vesiculoviurs } \\
\text { Coronavirus } \\
\text { Enterovirus } \\
\text { Alphavirus } \\
\text { Lentivirus }\end{array}$ & $\begin{array}{l}\text { Replication-deficient vectors based on Sendai virus } \\
\text { Live mumps virus in experimental settings and in a clinical trial to treat ovarian cancer. } \\
\text { Non-oncolytic replication-competent vectors based on simian virus } 5 . \\
\text { Live attenuated vesicular stomatitis virus and recombinant derivatives. } \\
\text { In vitro oncolytic activity of retargeted replication-competent vectors based on feline } \\
\text { coronavirus and murine hepatitis virus. } \\
\text { Live echovirus type } 1 . \\
\text { Live attenuated poliovirus and bovine enterovirus in preclinical testing } \\
\text { Both replication-deficient and replication-competent MoMLV } \\
\text { vectors also used clinical trials. } \\
\text { Replication-deficient vectors based on HIV-1. }\end{array}$ \\
\hline
\end{tabular}

Table 1: Summary on members of virus families that are evaluated in cancer gene therapy [14].

Reovirus permissiveness has been shown to correlate with the activation status of the Ras signaling pathway(s) in the host cell. Although Ras mutations are present in only about $30 \%$ of all human cancers, the fact that mutations in upstream activators and/or downstream effectors of Ras can also result in enhanced Ras signaling suggests that most cancers are theoretically treatable by reovirus [23]. Ras proteins function as molecular switches in many cellular processes including apoptosis, cell cycle transitions, protein translation, cytoskeletal rearrangement, and intracellular vesicle transport.

The cellular changes undergone by a cancer cell or induced by Ras transformation have favorable effects on multiple steps of reovirus replication. First, once the virus enters a cell it must undergo an uncoating step in order to initiate the infectious process. This step requires sufficient levels of cathepsin lysosomal proteases that are upregulated in cancer cells and by Ras signaling. Second, Ras-mediated transformation lends a favorable cellular environment that enhances infectivity of the progeny virus particles, possibly by facilitating post- translational modification of viral proteins, and their subsequent assembly into virions [24].

Cancer cells are significantly more sensitive to reovirus-induced apoptosis at late stages of the infection, resulting in enhanced release, and therefore spread of the virus through subsequent rounds of infection. The cumulative effect of these cellular changes results in the heightened sensitivity of cancer cells to reovirus infection, which thus far appears to also manifest in breast CICs [25].

Vesicular stomatitis virus: VSV, a member of the rhabdoviridae family, is a negative sense single-stranded RNA virus that is highly sensitive to the antiviral IFN response. This acute sensitivity to IFNinduced changes effectively blocks infection of the virus in normal cells. It also makes the virus an ideal naturally oncolytic agent as cancer cells often have a deregulated IFN response, allowing for uninhibited VSV replication. In addition, tumor cells with defects in Ras signaling pathway have been shown to be permissive to VSV [26]. 
VSV is an animal pathogen, which can also infect humans. In humans, VSV infections are usually asymptomatic, but it can cause flulike symptoms, raising some concern about using the virus as a cancer therapeutic in its naturally isolated state. Because the original observations that a laboratory VSV isolate efficiently infects and kills tumor cells in vitro and in vivo, natural mutants and engineered versions have been isolated with improved oncolytic cancer-killing capabilities and tumor specificity [27].

Different findings indicates that engineered versions such as recombinant VSV, M51, which has reduced toxicity but is highly effective at targeting various primary and metastasized tumors in vivo [28].

One other notable characteristic of VSV is its ability to encode for a matrix (M) protein. Once translated, the $M$ protein is capable of localizing to the nuclear pores of the host cell to block transcripts for immune system factors (e.g., cytokines, interferons) from accessing the cytoplasm. This not only prevents an antiviral response from mounting, but also induces cellular apoptosis resulting in VSV pathogenesis. The M-protein has also been studied for its ability to contribute towards viral budding and virion assembly [29].

Herpes Simplex Virus: HSV-1 is a unique virus that offers promise in treating several pediatric cancers including brain tumors, neuroblastomas, and sarcomas. The virus can be employed as oncolytic viral therapy, a direct, targeted attack or via gene therapy in which foreign genes are expressed therapeutically in cancer cells [30].

Genetically engineered HSV-1 has a number of advantages in the treatment of a subset of pediatric malignancies. HSV-1 is a large (152 $\mathrm{kb}, 89$ genes, multiple open-reading frames), double stranded DNA, enveloped virus that does not integrate into host DNA. HSV is a neurotropic virus thus making cancers of neural origin, including brain tumors and neuroblastomas, ideal targets [31].

However, it is equally able to infect and kill cells from a variety of cancers including sarcomas, melanomas, colon, breast, lung, prostate, and hepatic tumors. Normal cells are spared, whereas tumor cells are targeted by deleting genes critical for viral replication in normal cells but not necessary in tumor cells such as the "neurovirulence" gene, $\gamma 134.5$. It has been estimated that up to $30 \mathrm{~kb}$ of the HSV genome is nonessential for replication in tumor cells and, therefore, can be replaced with foreign DNA for gene therapy without affecting the virus's ability to infect tumor cells and replicate. From a safety standpoint, antiviral agents in clinical use are readily available in the unlikely event that the mutant HSV produces toxicity to normal tissues30 Replication-competent HSV vectors were one of the first viruses developed for this strategy and the ability of such genetically engineered viruses is that the viral replication and cytotoxicity are restricted to tumor cells but not to normal cells. Oncolytic HSV vectors have been tested in a variety of different tumor types and models, and found to be highly effective and safe [30].

Newcastle Disease Virus: NDV is a single-stranded RNA virus that is contagious to birds causing fatal sickness in most avian species. In humans, it is associated with minimal pathology comprised of mild conjunctivitis and laryngitis. The ability of NDV to directly replicate in and kill a variety of cultured human and rat neuroblastoma cells without effect in normal fibroblasts has been previously described [31].

The selective killing of human neuroblastoma cells was maintained during the in vivo treatment of established tumors in athymic nude mice. The strains HuJ and MTH-68 of NDV have been patented for their use as antineoplastic agents. Testing of the NDV strain MTH68 in patients with high grade glioma commenced in 1996 [32].

Csatary [32] reported that both pediatric and adult patients suffering from high grade glioma, and anecdotal evidence of efficacy in some patients was noted. Elsewhere a case report of a fourteen year old patient suffering from recurrent GBM was treated by IV administration of NDV. The last published report of this patient indicated that he was taking only NDV vaccine treatment and was living a relatively normal life. The NDV strain $\mathrm{HuJ}$ was tested for safety and efficacy in a phase trials and Intravenous administration of NDV was well tolerated and maximum tolerable dose (MTD) was not reached in this study [32].

Mechanism of action: There are several different effective mechanisms by which oncolytic viruses destroy malignant cells. Various mechanisms were proposed to account for the effects of viral infection on cancer. These include:

I. Direct tumor cell killing as a consequence of intracellular virus replication in malignant cells (direct virus oncolysis);

II. Immunologic reactions of cancer cells carrying persistent, nonlytic infections, apoptosis (indirect viral oncolysis);

III. Immunologic reactions of the host against tumor material released through viral cytolysis or post-oncolytic immunity, and

IV. Cell lysis and the expulsion of numerous progeny viral particles which can subsequently infect additional surrounding malignant cells, thereby enabling further lytic destruction within the tumor microenvironment [33].

Oncolytic viral therapy relies on virus replication in infected tumor cells that die and release infectious virus. In gene therapy, expression of therapeutic foreign gene products either directly or indirectly leads to cell death [32].

The virus itself can destroy tumor cells by replicating. This cycle then can repeat, by infection of adjacent cells and their subsequent destruction by the same mechanism.

Adenoviruses express E1A protein during replication, which mediates killing of tumor cells by increasing their sensitivity to tumor necrosis factor (TNF). Induction of specific anti-tumor immunity might result in long-term defense against cancer recurrence. Viral peptides are presented on the cell surface with MHC class 1 proteins; this complex is recognized by cytotoxic T lymphocytes (CTLs), which are attracted to the virally-transduced tumor cell. These CTLs then acquire specificity for cancer-specific antigens and kill the cells by a still unknown mechanism. In some animal models, systemic, specific antitumor immune responses have been demonstrated with a HSV virus type 1 mutant [29].

In addition, oncolytic viruses may induce immunotherapy, involving the substantially more complicated mechanism of oncolytic viral killing of malignant cells via the stimulation of the host immune system. In general, the immune system plays the natural role of immune surveillance via the detection of Tumor Associated Antigens (TAAs) and the elimination of neoplastic cells prior to tumor development [34].

Oncolytic viruses can contribute to this process through the direct lysis of malignant cells and the subsequent presentation of TAAs to the immune system. The viruses themselves are capable of evoking strong immune responses. Such a theory is behind the utilization of 
Page 5 of 6

oncolysates for therapy. Oncolysates, comprising of tumor cells that have been infected ex vivo, when administered, elicit strong antiviral and anti-tumoral immune responses and have shown promise in both animal models and human clinical trials [35].

Furthermore, some oncolytic viruses are modified to encode immune-stimulatory cytokines, including interleukin (IL)-12 and granulocyte macrophage-colony stimulating factor (GM-CSF) encoding HSVs (BioVex), adenoviruses (Cell Genesys) (Table 2).

\begin{tabular}{|l|l|}
\hline Mechanism & Examples \\
\hline Direct cell lysis due to viral replication & Adenoviruses Herpes simplex viruses \\
\hline Direct cytotoxicity of viral protein & Adenovirus E4ORF4 \\
\hline $\begin{array}{l}\text { Induction of antitumoral immunity } \\
\text { *Nonspecific (e.g., TNF) } \\
\text { *Specific (e.g., CTL response) }\end{array}$ & $\begin{array}{l}\text { Adenovirus (E1A) } \\
\text { Herpes simplex virus }\end{array}$ \\
\hline $\begin{array}{l}\text { Sensitization to chemotherapy and } \\
\text { radiation therapy }\end{array}$ & $\begin{array}{l}\text { Adenovirus (E1A) } \\
\text { Adenovirus (AdTK-RC) }\end{array}$ \\
\hline Transgene expression & $\begin{array}{l}\text { Herpes simplex virus (rRp450) } \\
\text { Vaccinia virus (GM-CSF) }\end{array}$ \\
\hline
\end{tabular}

Table 2: Summary on the mechanisms of antitumoral efficacy of oncolytic viruses [35].

Limitations of oncolytic viruses in curing cancer: Although several viruses have been shown to possess oncolytic abilities, there are still many unanswered questions and problems that need to be addressed. Getting viruses to the site of the tumour has been problematic since most experiments require injecting high viral titers directly into the tumour site. The efficacy of eliminating metastasized cancer by using oncolytic therapy may be very low since all cancerous cells must be removed to prevent relapses. Systemic delivery of oncolytic viruses via IV injection is difficult because of viral tropism and activation of the immune system upon viremia. Getting the virus into specific tumour areas is the challenge that lies ahead. The fear of oncolytic viruses evolving into pathogens is another concern that is deeply rooted in the belief that viruses are harmful [36].

The human body can place selective pressures on these viruses, but clinical trials for oncolytic viral therapy are designed to ensure that patients do not inadvertently infect other individuals. By preventing transmission or "serial passage" of oncolytic viruses, the probability of either mutating or recombining, and eventually reverting into its original pathogenic form, is reduced. Occasionally, administration of an oncolytic virus might cause an adverse response. Although this often is not lethal, one method of administration that has been used in some experiments is to gradually increase viral dosage over the course of several injections. Though adverse symptoms may appear after the first inoculation, the host will eventually become desensitized and the virus can continue its anti-tumour activity unimpeded by the immune system [36].

Further complicating matters are the ethical and economic factors that need to be considered with regards to oncolytic therapy. This raises some ethical concerns regarding practices in recruiting test subjects. However, it is economically feasible to relocate since the bulk of their market resides in some countries, where oncolytic therapies seem to earn government approval faster. The economic perspective is an important consideration since it has become extremely costly for biotechnology companies to research, produce, test and market these products. To date, only a handful of oncolytic viruses have made it into the advanced stages of FDA testing (e.g., human test trials), only to be withdrawn or rejected [37].

On the other hand, significant obstacles towards the application of safe and efficacious viral therapies have become apparent. These frequently relate to the lack of cell culture and animal tumor models that accurately reflect the characteristics of cancerous tissues in patients. Viral agents administered IV can be particularly effective against metastatic cancers, which are especially difficult to treat conventionally. However, blood-borne viruses can be deactivated by antibodies and cleared from the blood stream quickly e.g. by Kupffer cells (extremely active phagocytic cells in the liver, which are responsible for adenovirus clearance). Avoidance of the immune system until the tumour is destroyed could be the biggest obstacle to the success of oncolytic virus therapy [38].

\section{Conclusion and Recommendations}

The field of oncolytic virotherapy is expanding and viruses continue to hold promise as effective treatments in combination with chemotherapy or other therapeutic modalities. The identification, isolation, and characterization of such cells are likely to be paramount to discover effective new cancer therapies that prevent relapse and improve long-term overall survival. Because they are not subjected to the same mechanisms mediating resistance to cytotoxic chemotherapy and irradiation, there is ample reason to postulate oncolytic viruses will be effective at eradicating CICs. Recent advances in our understanding of tumor biology and virology have helped to overcome some of these hurdles, and different groups have successfully targeted features that varied from virus delivery to altering the host immune response. It is hoped that this collective efforts will finally pave way for the development of effective and safe viruses for cancer therapy. The challenge in creating the ideal oncolytic virus lies in finding the proper balance between two competing requirements. The various clinical trials using both in vitro and in vivo to treat benign and malignant tumor cells in human beings are not currently surely satisfactory because of some factors that hindered the curing efficiency.

Based on the above concluding remarks the following recommendations are forwarded:

Understanding of the mechanisms of viral oncolysis and the immune responses that viruses induce and the ability to image these viruses in vivo should be improved in order to make this therapeutic approach safer and more effective.

Genetically Modified oncolytic viruses must be applied to enhance their killing efficiency.

Various mechanisms must be designed to decrease the side effects, like toxicity, of oncolytic viral administration.

Recent technologies for viral gene therapy with feasible costs should be applicable.

Oncolytic viruses are administered in combination with standard chemotherapeutic regimes and immune-suppressive agents.

Detailed and promising trails on the oncolytic viral treatment must be conducted to cure tumor in animals. 


\section{Acknowledgements}

I would like to express my sincere appreciation to all of the journals which are of used as a good source of information to write this review.

\section{Conflict of Interest}

Conflict of interest declared none.

\section{References}

1. Donnenberg VS, Donnenberg AD (2005) Multiple drug resistance in cancer revisited: the cancer stem cell hypothesis. J Clin Pharmacol 45: 872-877.

2. Jordan CT (2009) Cancer stem cells: controversial or just misunderstood? Cell Stem Cell 4: 203-205.

3. Liu TC, Kirn D (2008) Gene therapy progress and prospects cancer: oncolytic viruses. Gene Ther 15: 877-884.

4. Islam MO, Kanemura Y, Tajria J, Mori H, Kobayashi S, et al. (2005) Functional expression of ABCG2 transporter in human neural progenitor cells. Neurosci Res 52: 75.

5. Aghi M, Rabkin S, Martuza RL (2006) Effect of chemotherapy-induced DNA repair on oncolytic herpes simplex viral replication. J Natl Cancer Inst 98: 38-50.

6. Kelly E, Russell SJ (2007) History of oncolytic viruses: genesis to genetic engineering. Mol Ther 15: 651-659.

7. Redding N, Zhou HY, Lun X, Senger D, Forsyth P, et al. (2009) The utility of oncolytic viruses against neuroblastoma. In The 5th International Meeting on Replicating Oncolytic Virus Therapeutics, Banff, Canada.

8. Lichty BD, Power AT, Stojdl DF, Bell JC (2004) Vesicular stomatitis virus: re-inventing the bullet. Trends Mol Med 10: 210-216.

9. Liu TC, Galanis E, Kirn D (2007) Clinical trial results with oncolytic virotherapy: a century of promise, a decade of progress. Nat Clin Pract Oncol 4: 101-117.

10. Mullen JT, Tanabe KK (2002) Viral oncolysis. Oncologist 7: 106-119.

11. Sinkovics JG, Horvath JC (2008) Natural and genetically engineered viral agents for oncolysis and gene therapy of human cancers. Arch Immunol Ther Exp (Warsz) 56 Suppl 1: 3s-59s.

12. Terada K, Wakimoto H, Tyminski E, Chiocca EA, Saeki Y (2006) Development of a rapid method to generate multiple oncolytic HSV vectors and their in vivo evaluation using syngeneic mouse tumor models. Gene Ther 13: 705-714.

13. Bauerschmitz GJ, Ranki T, Kangasniemi L, Ribacka C, Eriksson M, et al. (2008) Tissue-specific promoters active in CD44+CD24-/low breast cancer cells. Cancer Res 68: 5533-5539.

14. Vähä-Koskela MJ, Heikkilä JE, Hinkkanen AE (2007) Oncolytic viruses in cancer therapy. Cancer Lett 254: 178-216.

15. Fueyo J, Gomez-Manzano C, Alemany R, Lee PS, McDonnell TJ, et al. (2000) A mutant oncolytic adenovirus targeting the $\mathrm{Rb}$ pathway produces anti-glioma effect in vivo. Oncogene 19: 2-12.

16. Ribacka C, Hemminki A (2008) Virotherapy as an approach against cancer stem cells. Curr Gene Ther 8: 88-96.

17. Eriksson M, Guse K, Bauerschmitz G, Virkkunen P, Tarkkanen M, et al. (2007) Oncolytic adenoviruses kill breast cancer initiating CD44+CD24-/low cells. Mol Ther 15: 2088-2093.

18. Ponti D, Costa A, Zaffaroni N, Pratesi G, Petrangolini G, et al. (2005) Isolation and in vitro propagation of tumorigenic breast cancer cells with stem/progenitor cell properties. Cancer Res 65: 5506-5511.

19. Alain T, Hirasawa K, Pon KJ, Nishikawa SG, Urbanski SJ, et al. (2002) Reovirus therapy of lymphoid malignancies. Blood 100: 4146-4153.
20. Yap TA, Brunetto A, Pandha H, Harrington K, Debono JS (2008) Reovirus therapy in cancer: has the orphan virus found a home. Expert Opin Investig Drugs 17: 1925-1935.

21. Marcato P, Dean CA, Giacomantonio CA, Lee PW (2009) Oncolytic reovirus effectively targets breast cancer stem cells. Mol Ther 17: 972-979.

22. Bao S, Wu Q, McLendon RE, Hao Y, Shi Q, et al. (2006) Glioma stem cells promote radioresistance by preferential activation of the DNA damage response. Nature 444: 756-760.

23. Strong JE, Coffey MC, Tang D, Sabinin P, Lee PW (1998) The molecular basis of viral oncolysis: usurpation of the Ras signaling pathway by reovirus. EMBO J 17: 3351-3362.

24. Golden JW, Linke J, Schmechel S, Thoemke K, Schiff LA (2002) Addition of exogenous protease facilitates reovirus infection in many restrictive cells. J Virol 76: 7430-7443.

25. Pruitt K, Pruitt WM, Bilter GK, Westwick JK, Der CJ (2002) Rafindependent deregulation of $\mathrm{p} 38$ and JNK mitogen-activated protein kinases are critical for Ras transformation. J Biol Chem 277: 31808-31817.

26. Balachandran S, Porosnicu M, Barber GN (2001) Oncolytic activity of vesicular stomatitis virus is effective against tumors exhibiting aberrant p53, Ras, or myc function and involves the induction of apoptosis. J Virol 75: 3474-3479.

27. Lun X, Senger DL, Alain T, Oprea A, Parato K, et al. (2006) Effects of intravenously administered recombinant vesicular stomatitis virus (VSV (deltaM51)) on multifocal and invasive gliomas. J Natl Cancer Inst 98: 1546-1557.

28. Stanford MM, McFadden G (2007) Myxoma virus and oncolytic virotherapy: a new biologic weapon in the war against cancer. Expert Opin Biol Ther 7: 1415-1425.

29. Stojdl DF, Lichty B, Knowles S, Marius R, Atkins H, et al. (2000) Exploiting tumor-specific defects in the interferon pathway with a previously unknown oncolytic virus. Nat Med 6: 821-825.

30. Tollefson AE, Ryerse JS, Scaria A, Hermiston TW, Wold WS (1996) The E3-11.6-kDa adenovirus death protein (ADP) is required for efficient cell death: characterization of cells infected with adp mutants. Virology 220: 152-162.

31. Lorence RM, Reichard KW, Katubig BB (1994) Complete regression of human neuroblastoma xenografts in athymic mice after local Newcastle disease virus therapy. J Natl Cancer Inst 86: 1228-1233.

32. Csatary LK, Bakács T (1999) Use of Newcastle disease virus vaccine (MTH-68/H) in a patient with high-grade glioblastoma. JAMA 281: 1588-1589.

33. Whyte P, Buchkovich KJ, Horowitz JM, Friend SH, Raybuck M, et al. (1988) Association between an oncogene and an anti-oncogene: the adenovirus E1A proteins bind to the retinoblastoma gene product. Nature 334: 124-129.

34. Stojdl DF, Lichty BD, TenOever BR, Paterson JM, Power AT (2003) VSV strains with defects in their ability to shutdown innate immunity are potent systemic anti-cancer agents. Cancer Cell 4: 263-275.

35. Marcato P, Shmulevitz M, Pan D, Stoltz D, Lee PW (2007) Ras transformation mediates reovirus oncolysis by enhancing virus uncoating, particle infectivity, and apoptosis-dependent release. Mol Ther 15: 1522-1530.

36. Bell JC, Lichty B, Stojdl D (2003) Getting oncolytic virus therapies off the ground. Cancer Cell 4: 7-11.

37. Garber K (2006) China approves world's first oncolytic virus therapy for cancer treatment. J Natl Cancer Inst 98: 298-300.

38. Freeman AI, Zakay-Rones Z, Gomori JM, Linetsky E, Rasooly L, et al. (2006) Phase I/II trial of intravenous NDV-HUJ oncolytic virus in recurrent glioblastoma multiforme. Mol Ther 13: 221-228. 